\title{
Calculation of the Absorption Spectrum of Benzene in Condensed Phase. A Study of the Solvent Effects
}

\author{
KALINE COUTINHO, ${ }^{1}$ SYLVIO CANUTO, ${ }^{1}$ M. C. ZERNER $^{2}$ \\ ${ }^{1}$ Instituto de Física, Universidade de São Paulo, CP 66318, 05315-970 São Paulo, SP, Brazil \\ ${ }^{2}$ Quantum Theory Project, University of Florida, Gainesville, Florida 32611
}

Received 3 March 1997; revised 23 April 1997; accepted 29 April 1997

\begin{abstract}
The red shift of the first absorption band of benzene in the condensed phase is calculated. The solid-phase calculations are performed using the experimental crystal structure. For the liquid two different approaches are considered. One is the herringbone structure suggested by X-ray experiments, and the other uses supermolecules generated by Monte Carlo simulation. Attention is given to the contribution of the usual parallel and T-shape conformations of the benzene dimers obtained in the simulation. Comparison with experiment shows a relatively poor result for the herringbone structure and good agreement for the supermolecular structures generated by the simulation. (c) 1997 John Wiley \& Sons, Inc. Int J Quant Chem 65: 885-891, 1997
\end{abstract}

\section{Introduction}

$\mathrm{n}$ this study we calculate and analyze the red shift of the first absorption band of benzene in the condensed phase as compared to the gas phase. The interest in the structure [1] and spectra of clusters and condensed-phase benzene is related to several topics of interest in chemistry, physics, and biology. It naturally relates to the importance of the interaction between aromatic $\pi$-systems. This present study also relates to our interest in solvent

Correspondence to: S. Canuto.

Contract grant sponsors: CNPq, FAPESP, and Office of Naval Research. effects in molecular absorption spectroscopy $[2,3]$. For the calculation of the solvatochromic shift of liquid benzene, we use two structures. One is the well-known herringbone structure [4] and the other is a disordered structure obtained from Monte Carlo (MC) simulation. For completeness, we also consider the first absorption transition of the solid benzene. All quantum mechanical calculations are made using the INDO/CIS model [5] as implemented in the ZINDO program [6], and we use the so-called supermolecular (SM) approach. In the case of the solid and the herringbone structure of the liquid, two configurations are used (Fig. 1). In the first we use 14 benzene molecules both for the solid and the liquid, as seen in Figure 1(a) (top). In the second, we use only 13 benzene molecules. 


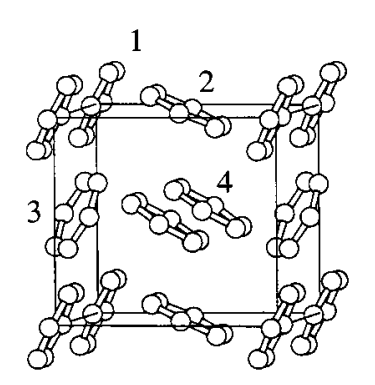

(a)
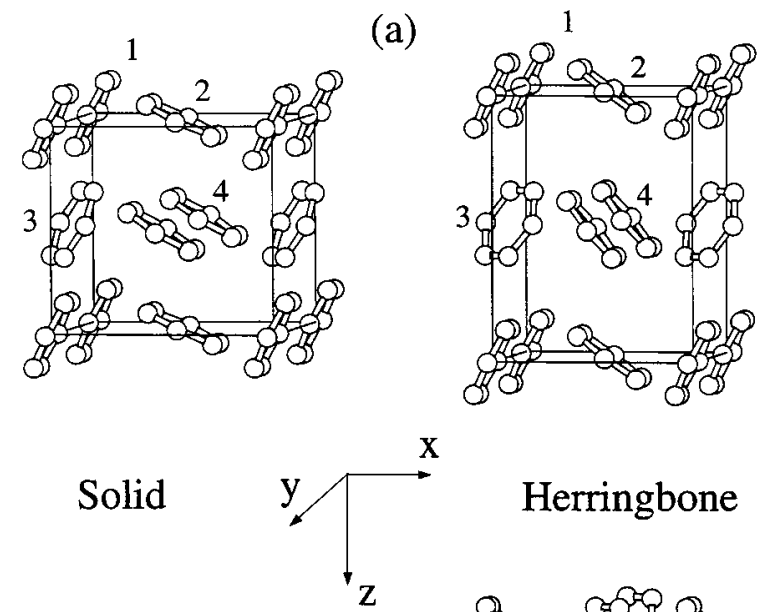

$\mathrm{X}$

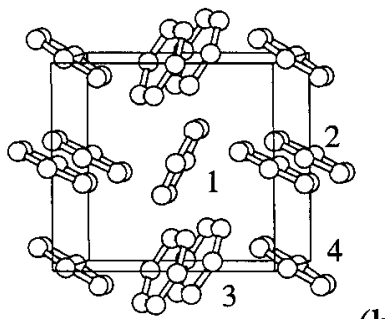

(b)

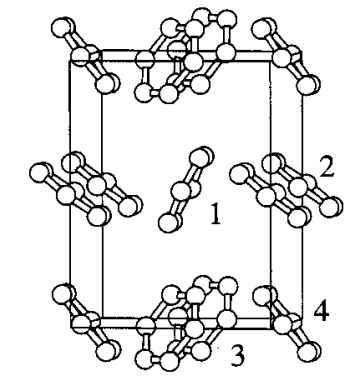

FIGURE 1. Crystalline and the herringbone structures of solid and liquid benzene. The top figures show 14 benzene molecules. The bottom figures show instead 13 molecules and includes one bulk benzene in the center. Molecules 1, 2, 3, and 4 generate the unit cell as defined by Narten [4].

This corresponds to including one benzene and its 12 nearest neighbors of the well-known $\mathrm{P} b c a$ space group [4]. This second case, as seen in Figure 1(b) (bottom), as opposed to the first case, includes a central benzene molecule to represent the "bulk." Calculations are then performed at these four structures but in each case using a fixed geometry.

For the case of the disordered liquid benzene the structures generated by the MC simulation [7] are used. Each SM is composed of 14 benzene molecules. As we shall see, this corresponds to including the first coordination shell as obtained from the radial distribution function (RDF). In addition, however, several SM calculations are performed. The actual number of SM structures used takes into account the statistical correlation between MC structures. The final excitation energy is obtained averaging over the transition energies obtained for each structure. A comparison of these different procedures will be made.

\section{Monte Carlo Simulation}

The MC simulation is performed using standard procedures for the Metropolis sampling technique [8] in the canonical ensemble, where the number of molecules $N$, the volume $V$, and the temperature $T$ are fixed. As usual, periodic boundary conditions in a cubic box [9] are used. In our simulation, we use 125 molecules of benzene. The volume of the cubic box is determined by the experimental density of the liquid, $\rho=0.8990 \mathrm{~g} / \mathrm{cm}^{3}$ [10], and the temperature is $T=25^{\circ} \mathrm{C}$. There has been much discussion about the quadrupolar interaction of benzene and its relation to the structure [11] (see next section). In our theoretical experiments, we are willing to generate a disordered structure for the liquid. Therefore, the benzene molecules interact only by the Lennard-Jones potential:

$$
U_{a b}=\sum_{i}^{\text {on } a \text { on } b} \sum_{j} 4 \varepsilon_{i j}\left[\left(\frac{\sigma_{i j}}{r_{i j}}\right)^{12}-\left(\frac{\sigma_{i j}}{r_{i j}}\right)^{6}\right] \text {, }
$$

where $\sum^{a}$ is the sum over the atoms of molecule $a$, $\sum^{b}$ is the sum over the atoms of molecule $b, r_{i j}$ is the distance between atoms $i$ and $j, \varepsilon_{i j}=\sqrt{\varepsilon_{i} \varepsilon_{j}}$, $\sigma_{i j}=\sqrt{\sigma_{i} \sigma_{j}}$, and $\sigma_{i}$ and $\varepsilon_{i}$ are the parameters of atom $i$. The potential parameters used were developed by Jorgensen and co-workers [12]: $\sigma_{\mathrm{C}}=3.75$ $\AA, \varepsilon_{C}=0.11 \mathrm{kcal} / \mathrm{mol}$, and $\sigma_{H}=\varepsilon_{H}=0$. As the environment of the liquid system has no significant effect on the conformational equilibrium for the benzene molecule $[4,14]$, we consider, in the simulation, rigid conformations of the benzene molecules in the $D_{6 h}$ structure with $r_{\mathrm{CC}}=1.40 \AA$, $r_{\mathrm{CH}}=1.088 \AA$, and all angles $\theta=120^{\circ}$. We recognize that distortions of the benzene structure would lead to considerably enhanced intensity of the forbidden ${ }^{1} B_{2 u}$ transition we examine. We do not believe, however, that the position of the band maximum will be much effected [15].

In the calculation of the pairwise energy, each molecule interacts with all other molecules within a center-of-mass separation that is smaller than the cutoff radius $r_{c}=13.11 \AA$. For separations larger than $r_{c}$, we use the long-range correction of the potential energy [13]. Over the 125 benzene molecules included in the simulation, each molecule interacts effectively with $\sim 60$ neighbors.

The initial configuration is generated randomly, considering the position and the orientation of 
each molecule. A new configuration, or one MC step, is generated by selecting all molecules sequentially and trying to translate it in all the Cartesian directions and also rotate it around a randomly chosen axis. The maximum allowed displacement of the molecules is auto-adjusted after $50 \mathrm{MC}$ steps to give an acceptance rate of new configurations around $50 \%$. The maximum rotation angle is fixed during the simulation in $\delta \theta=$ $\pm 15^{\circ}$. The full simulation [7] involves a thermalization stage of about $5000 \mathrm{MC}$ steps followed by an averaging stage of $48,000 \mathrm{MC}$ steps. During the averaging stage some thermodynamical properties, as the internal energy and the heat capacity at constant volume, are calculated, and they are in complete agreement with that calculated by Jorgensen and co-workers [12]. The radial distribution function is also calculated during the averaging stage in the simulation.

\section{Results and Discussions}

The structure of the liquid benzene has been of interest for many years. Ever since the X-ray study of Narten [4], the herringbone structure is believed to prevail. This structure is illustrated in Figure 1 along with the known experimental structure of solid benzene. In the case of the benzene dimer the interest is basically related to the relative stabilization of the parallel and T-shape structure [16]. A herringbone structure for the dimer has also been proposed [17]. More recent studies [18] have indicated that a parallel-displaced configuration seems to be more stable than the T-shape favorite structure for the dimer. It seems clear from all these studies that the parallel-displaced, T-shape, and T-shape-displaced structures are very close in energy.

From these considerations it seems appropriate to consider SM calculations of the liquid benzene with all these possibilities, not only the herringbone structure. Thus several structures generated by the MC simulation will be used. As these structures are statistically correlated, we will select structures that are separated by a certain number of MC steps. This number results from the time correlation function and the statistical inefficiency analysis [19]. In fact, we select structures that are separated by twice the correlation time $(2 \mathscr{T})$, obtained after the integration of the time correlation function. Clearly, "time" here should mean MC step. The calculated time correlation function, $C(t)$, and the statistical inefficiency, $s$, for the energies of the liquid benzene structures are shown in Figure 2. The calculated $C(t)$ function gives the correlation time value of $\mathscr{T} \sim 400$ steps. This is related to the statistical inefficiency and the relation $s \approx 2 \mathscr{T}$ holds. Thus we use only MC structures that are separated by $800 \mathrm{MC}$ steps. As the entire simulation took $48,000 \mathrm{MC}$ steps, only 60 are statistically relevant and used in our studies.

Figure 3 shows a comparison between the calculated RDF from the MC simulation and the experimental RDF of Narten [4]. As can be seen, the overall agreement between these two results is very good. The results show a first broad peak ranging from 3.0 to $7.6 \AA$. Integration of this peak gives the coordination number of $N_{s}=12.5$. This theoretical result is of interest in two different aspects. First, the experimental result of Narten gives $N_{s}=12$, in close agreement with our theoretical value. This suggests that $N_{s}$ is not enough to uniquely characterize the liquid as having a herringbone structure. The second aspect is of more technical interest. $N_{s}=12.5$ signifies that, on the average, each benzene molecule has 13 (rounding off) neighbors and therefore the supermolecule should have 14 molecules. Hence all three systems, including the solid and the herringbone, will be calculated with the same number of molecules. The difference is, however, that in the case of the MC liquid a total of $60 \mathrm{SM}$ quantum mechanical calculations are performed.

In all cases the excitation energy to the $B_{2 u}(\pi-$ $\left.\pi^{*}\right)$ state of benzene is calculated including all the $e_{1 g} \rightarrow e_{2 u}$ transitions of each individual benzene; that is, from 28 occupied molecular orbitals into 28 molecular orbitals of the virtual space.

Table I summarizes the calculated numerical results and compares them with the experimental values for the red shift of benzene in condensed phase.

Figure 4 shows the calculated result for the excitation energy of the MC liquid together with the final average $\left(-306 \mathrm{~cm}^{-1}\right)$ and the experimental [20] $\left(-320 \mathrm{~cm}^{-1}\right)$ values for the shift compared to the gas phase. It should be noted that the shift for an individual structure may differ by a few hundreds of $\mathrm{cm}^{-1}$, but the "ensemble average" final result is in very good agreement with the experimental result. It is interesting to analyze, wherever possible, the contribution of the individual benzene molecules. This is, of course, not always possible. However, we note that in many 
COUTINHO, CANUTO, AND ZERNER

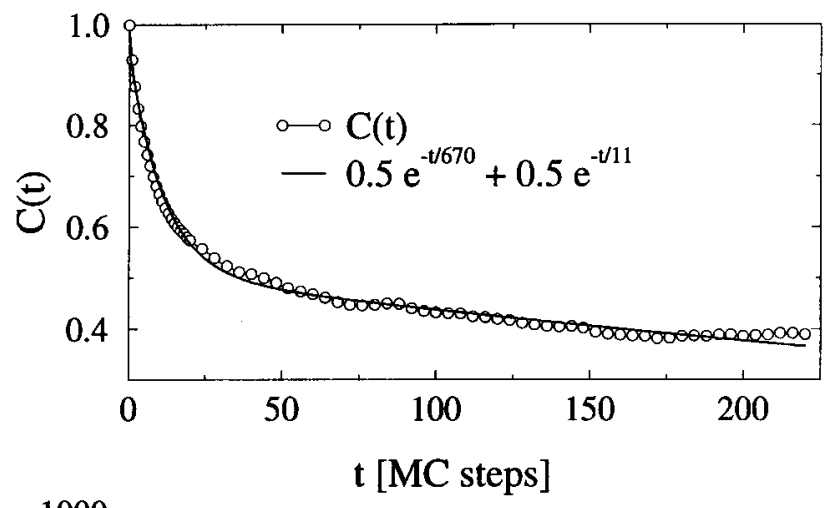

(a)

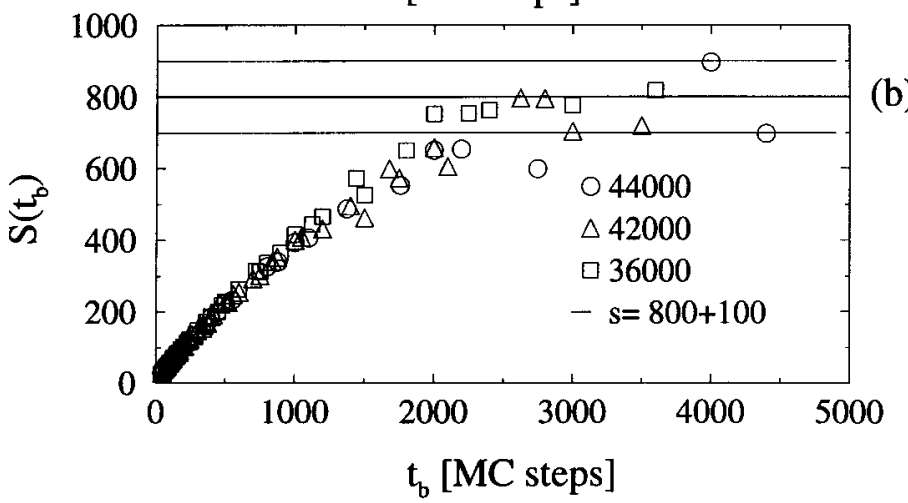

FIGURE 2. Time correlation function (top) and the statistical inefficiency (bottom) of the MC simulation. See text.

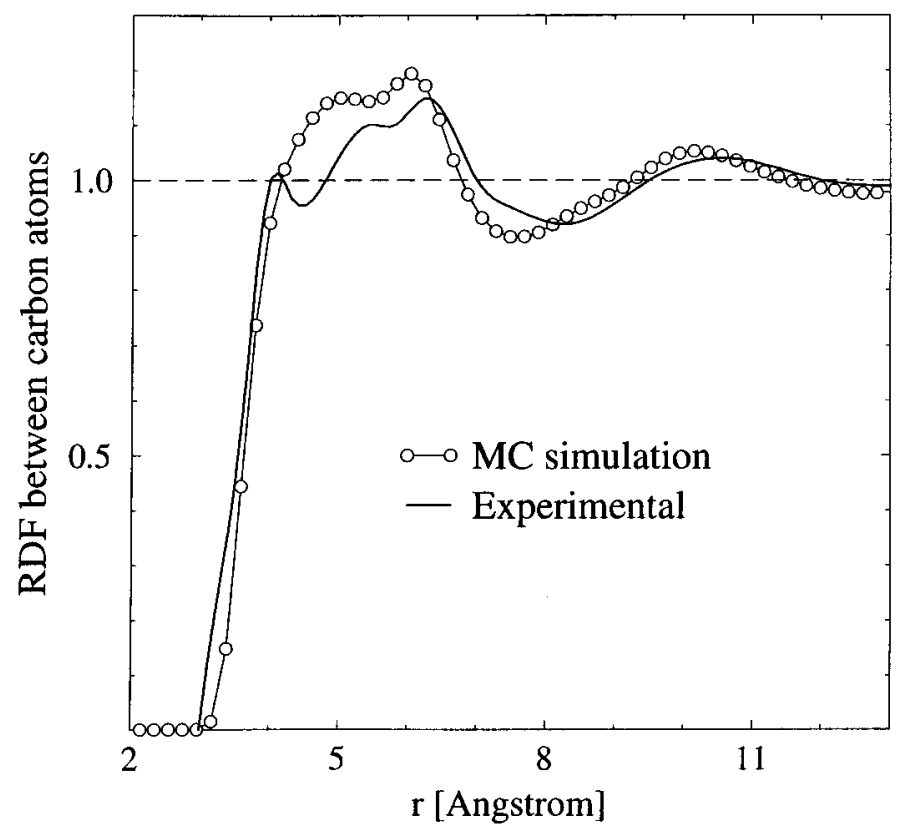

FIGURE 3. Comparison of the calculated and experimental [4] carbon-carbon radial distribution function of the liquid benzene. 
TABLE I

Summary of the calculated and experimental

results for the $B_{2 u}\left(\pi-\pi^{*}\right)$ energy shift $\left(\mathrm{cm}^{-1}\right)$

in the absorption spectra of benzene in

condensed phases. $^{\mathrm{a}}$

\begin{tabular}{lrrc}
\hline \multicolumn{1}{c}{ System } & $N$ & $\Delta E_{\text {calc. }}$ & $\Delta E_{\text {exp. [20] }}$ \\
\hline Gas & 1 & 0 & 0 \\
Solid $^{\mathrm{b}}$ & 13 & -260 & $-260 \pm 30$ \\
Liquid (herringbone) $^{\mathrm{c}}$ & 14 & -240 & \\
& 13 & -100 & $-320 \pm 30$ \\
Liquid (herringbone) $^{\mathrm{d}}$ & 14 & -60 & \\
& 13 & -100 & $-320 \pm 30$ \\
Liquid (MC) $^{\mathrm{e}}$ & 14 & -40 & \\
\hline
\end{tabular}

${ }^{\mathrm{a}} N$ is the number of molecules included in the quantum calculations.

${ }^{\text {b }}$ Lattice parameters [4] $(7.460,9.666,7.034)$ in $\AA$.

${ }^{c}$ Density of $0.874 \mathrm{~g} / \mathrm{cm}^{3}$ corresponding to the lattice parameters [4] $(6.75,9.74,9.03)$ in $\AA$.

${ }^{\mathrm{d}}$ Density of $0.899 \mathrm{~g} / \mathrm{cm}^{3}$ (used in the $\mathrm{MC}$ simulation) corresponding to the lattice parameters $(6.75,9.74,8.78)$ in $\AA$.

${ }^{\mathrm{e}}$ Average value over $60 \mathrm{SM}$ structures.

structures the transition is somewhat localized in two benzene molecules. Interesting enough, these two molecules are found to be in well-known conformations, like the T-shape-displaced and the parallel-displaced (see Fig. 5 for illustration). Therefore we now look at systems composed of

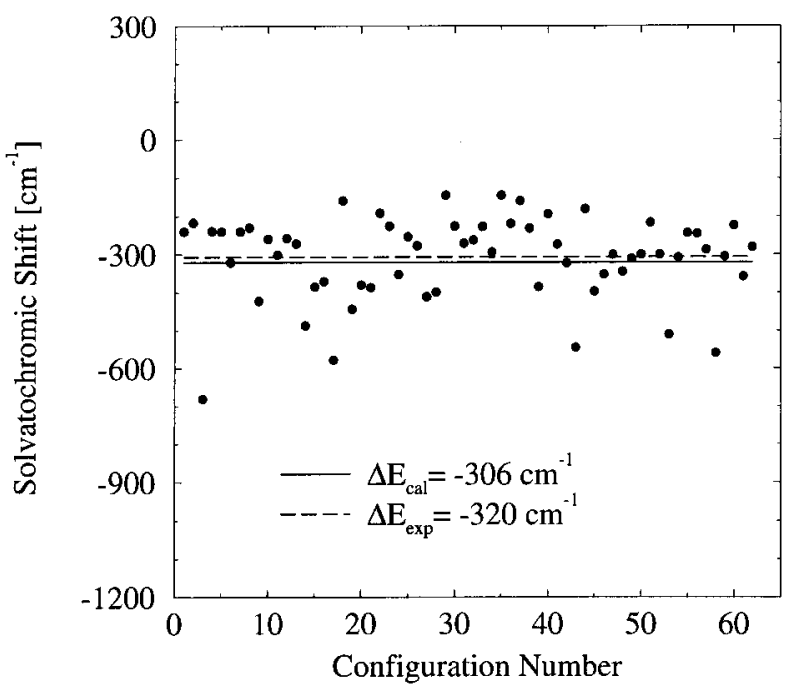

FIGURE 4. Calculated red shift obtained from the disordered MC structures of the liquid benzene. Also shown is the average value (solid line) and the experimental result (dashed line).
T-shape-displaced

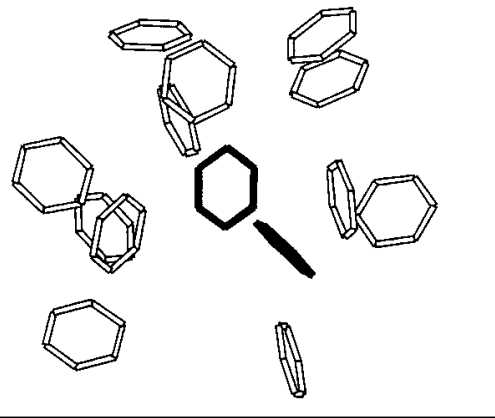

Parallel-displaced



FIGURE 5. Illustration of MC structures showing the T-shape, displaced, and parallel-displaced dimer conformations. These dimers give a large contribution to the total red shift. See text and Table II.

only two benzene molecules. We find from the MC simulation that $\sim 25 \%$ of the benzenes have the nearest neighbor within an angle between 0 and $20^{\circ}$, corresponding to the parallel and paralleldisplaced conformations of the dimer. Similarly, $\sim 15 \%$ have its nearest neighbor within an angle between 70 and $90^{\circ}$, corresponding to the T-shape, T-shape-displaced, and L-shape conformations. The average contribution of these "ordered" dimer conformations is compared, in Table II, with the result obtained using all dimers generated in the Monte Carlo simulation irrespective of the angle. Dimers alone are not able to give a good result for the red shift, but it is clear that those ordered dimer conformations make contributions that are significant. We note, in particular, that these parallel conformations give larger shifts than the perpendicular ones. This seems to suggest that these ordered dimer conformations of benzene are of importance in understanding the spectroscopic red shift of the liquid phase. 
TABLE II

Summary of the average contribution of different

dimer conformations to the calculated shift for

the $B_{2 u}\left(\pi-\pi^{*}\right)$ absorption transition of

liquid benzene. ${ }^{a}$

\begin{tabular}{lrc}
\hline \multicolumn{1}{c}{ System } & $N$ & $\Delta E_{\text {calc. }}\left(\mathrm{cm}^{-1}\right)$ \\
\hline MC T-shape & 2 & -98 \\
MC T-shape-displaced & 2 & -135 \\
$\quad$ and L-shape & & -179 \\
MC parallel & 2 & -197 \\
MC parallel-displaced & 2 & -103 \\
MC dimer & 2 & -306 \\
MC supermolecule & 14 & \\
\hline
\end{tabular}

${ }^{a} N$ is the number of molecules included in the quantum calculations.

In the case of the herringbone structure with 14 molecules, the calculated shift is too small, only $-60 \mathrm{~cm}^{-1}$. This discrepancy, however, is not found in the similar structure of the solid. In this case the calculated result is $-240 \mathrm{~cm}^{-1}$ in good agreement with the experimental value of $-260 \mathrm{~cm}^{-1}$ [20].

A possible deficiency of the use of the herringbone structure shown in Figure 1(a) is that there is no benzene molecule in the center of the box to represent the "bulk." To look at this we have used the structures shown in Figure 1(b) for the herringbone and the solid. The calculated results, however, changed only from the previous -60 to -100 $\mathrm{cm}^{-1}$ showing only slight improvement. For the solid it changed from the previous -240 to -260 $\mathrm{cm}^{-1}$, bringing the shift to a better agreement with the experimental result.

\section{Summary and Conclusions}

In this work we have calculated the red shift of the first absorption band of benzene in the condensed phase. The herringbone structure used for the liquid gives a red shift that is only $15-25 \%$ of the experimental value. In turn, the use of a disordered liquid obtained from Monte Carlo simulation gives a red shift that is in very good agreement with experiments. In this disordered liquid most of the usual structures of the benzene dimer, such as the parallel, parallel-displaced, T-shape, T-shape-displaced, etc., are included naturally, but there is little long-range order. The calculated red shift for crystalline benzene is also in very good agreement with the experimental shift.

\section{ACKNOWLEDGMENTS}

This work is supported in part by grants from $\mathrm{CNPq}$ and FAPESP and from the Office of Naval Research.

\section{References}

1. I. Pettersson and T. Liljefors, J. Comput. Chem. 8, 1139 (1987); N. L. Allinger and J. H. Lii, J. Comput. Chem. 8, 1146 (1987); X. Shi and L. S. Bartell, J. Phys. Chem. 92, 5667 (1988); L. S. Bartell, L. R. Sharkey, and X. Shi, J. Am. Chem. Soc. 110, 7006 (1988)

2. C. Reichardt, Solvents and Solvent Effects in Organic Chemistry (Verlag-Chemie, Weinheim, 1979); E. S. Amis and J. F. Hinton, Solvent Effects on Chemical Phenomena, Vol. 1 (Academic Press, New York, 1973); P. Beak, Acc. Chem Res. 10, 186 (1977); M. F. Nicol, Solvent Effects on Electronic Spectra, App. Spectr. Rev. 8, 183 (1974); N. S. Bayliss and E. G. McRae, J. Phys. Chem. 58, 1002 (1954); A. Warshel, Computer Modeling of Chemical Reactions in Enzymes and Solutions (Wiley, New York, 1991); J. Tomasi and M. Persico, Chem Rev. 94, 2027 (1994); C. J. Cramer and D. G. Truhlar, in Reviews in Computational Chemistry, Vol. 6, D. B. Boyd and K. B. Lipkowitz, Eds. (VCH, New York, 1995); J. Gao, Acc. Chem. Res. 29, 298 (1996).

3. K. Coutinho and S. Canuto, Adv. Quantum Chem. 28, 89 (1997); N. Rösch and M. C. Zerner, J. Phys. Chem. 98, 5817 (1994); M. Karelson and M. C. Zerner, J. Phys. Chem. 96, 6949 (1992); M. A. Thompson and M. C. Zerner, J. Am. Chem. Soc. 113, 8210 (1991).

4. A. H. Narten, J. Chem. Phys. 48 (1968) 1630; A. H. Narten, J. Chem. Phys. 67 (1977) 2102.

5. J. Ridley and M. C. Zerner, Theor. Chim. Acta 32, 111, (1973).

6. ZINDO: A semi-empirical program package, M. C. Zerner, University of Florida, Gainesville, FL 32611.

7. DICE: A Monte Carlo program for molecular liquid simulation, K. Coutinho and S. Canuto, University of São Paulo, Brazil.

8. N. Metropolis, A. W. Rosenbluth, M. N. Rosenbluth, A. H. Teller, J. Chem. Phys. 21, 1087 (1953).

9. M. P. Allen and D. J. Tildesley, Computer Simulation of Liquids (Clarendon Press, Boca Raton, FL, 1987).

10. CRC Handbook of Chemistry and Physics, 64th ed. (CRC Press, 1983-1984).

11. W. L. Jorgensen and D. L. Severance, J. Am. Chem. Soc. 112, 4768 (1990).

12. W. L. Jorgensen, J. Chandrasekhar, and J. D. Madura, J. Am. Chem. Soc. 106, 6638 (1984).

13. W. L. Jorgensen, R. C. Binring, Jr., and B. Bigot, J. Am. Chem. Soc. 103, 4393 (1981).

14. W. L. Jorgensen, J. Phys. Chem. 87, 5304 (1983).

15. G. Pearl, M. C. Zerner, A. Broo, and J. McKelvey, in progress.

16. K. C. Jandra, J. C. Hemminger, J. S. Winn, S. E. Novick, S. J. Harris, and W. J. Klemperer, J. Chem. Phys. 63, 1419 (1975); J. M. Steed, T. A. Dixon, and W. J. Klemperer, J. Chem. 
Phys. 70, 4940 (1979); G. Karlström, P. Linse, A. Wallqvist, and B. Jönsson, J. Am. Chem. Soc. 105, 3777 (1983); P. Hobza, H. L. Selzle, and E. W. Schlag, J. Chem. Phys. 93, 5893 (1990).

17. D. E. Williams, Acta Cryst. A36, 715 (1980); M. Schamer and E. R. Bernstein, J. Chem. Phys. 82, 3722 (1985).
18. P. Hobza, H. L. Selzle, and E. W. Schlag, J. Am. Chem. Soc. 116, 3500 (1994); P. Hobza, H. L. Selzle, and E. W. Schlag, Chem. Rev. 94, 1767 (1994).

19. K. Coutinho, M. J. de Oliveira, and S. Canuto, to appear.

20. T. Inagaki, J. Chem. Phys. 57 (1972) 2526; V. L. Broude, Sov. Phys. Usp. 4, 584 (1962). 